Mon. Not. R. Astron. Soc. 000, 177(2016) Printed 27 December $2018 \quad$ (MN LATEX style file v2.2)

\title{
Does PSR J1417-4402 system behave as a "Redback"?
}

\author{
M. A. De Vito ${ }^{1,2 \star}$, J. E. Horvath ${ }^{3} \dagger$, O. G. Benvenuto ${ }^{1,2} \ddagger$ \\ 1 Instituto de Astrofísica de La Plata, IALP, CCT-CONICET-UNLP, Argentina \\ ${ }^{2}$ Facultad de Ciencias Astronómicas y Geofísicas, Universidad Nacional de La Plata (UNLP), \\ Paseo del Bosque S/N, B1900FWA, La Plata, Argentina \\ 3 Instituto de Astronomía, Geofísica e Ciências Atmosféricas, Universidade de São Paulo, \\ R. do Matão 1226 (05508-090), Cidade Universitária, São Paulo SP, Brazil
}

December 19

\begin{abstract}
We study the present evolutionary status of the binary system containing the $2.66 \mathrm{~ms}$ pulsar PSR J1417-4402 in a 5.4 day orbit. This is the pulsar in the original source 3FGL J1417.5-4402, that has undergone a transition from X-ray state to a pulsar state, just like some redbacks did. The system has many characteristics similar to redback pulsars family, but is on a much wider orbit. We show that close binary evolution including irradiation feedback driven by the luminosity due to accretion onto the neutron star component of the pair, and evaporation due to pulsar emission, is able to account for the masses of the components and the photometric data of the donor star. The tracks leading to the present PSR J1417-4402 are degenerate within a range of parameters, suggesting that the same physics invoked to explain the redback/black widows groups leads to the formation of much wider orbit systems, outside the redback region limits.
\end{abstract}

Key words: (stars:) binaries (including multiple): close, stars: evolution, (stars:) pulsars: general, (stars:) pulsars: individual: PSR J1417-4402

\section{INTRODUCTION}

Millisecond pulsars (MSPs) are thought to be the final product of close binary systems (CBSs) evolution, in which a donor star transfers mass and angular momentum to its companion, an old neutron star (NS). The first member of a new class of intriguing objects was discovered by Fruchter, Stinebring, \& Taylor (1988). Since the data was interpreted as a fast NS ablating its companion, as a result of a former spin-up by accretion, the system was dubbed a "black widow" (BW). Since then on, this family of objects has grown very fast, and now they are part of a group usually refered as "spiders" 1. Isolated MSPs could result from the final stage of these systems in which evaporation of the

\footnotetext{
* Member of the Carrera del Investigador Científico, Consejo Nacional de Investigaciones Científicas y Técnicas (CONICET). Email: adevito@fcaglp.unlp.edu.ar † Email: foton@astro.iag.usp.br

$\ddagger$ Member of the Carrera del Investigador Científico, Comisión de Investigaciones Científicas de la Provincia de Buenos Aires (CIC). Email: obenvenu@fcaglp.unlp.edu.ar

1 We shall use the denominations "CBSs" and "spiders" interchangeably in this work, in spite that some details of these names are not exactly the same. Since our work deals with intermittent systems this subtelty is not important.
}

companion is complete. Later on, a group of accreting systems with comparable value of its orbital period $\left(P_{\text {orb }}\right)$, but much higher donor masses was identified, and more recently its number increased considerably due to Fermi/LAT data. A type of Australian spider ("redback", RB), the cousin to the North American black widow spider, are used to name this group. Both groups have $P_{\text {orb }}$ shorter than 1 day, although BW and RB companions have $M_{2} \lesssim 0.05 M_{\odot}$ and $0.1 M_{\odot} \lesssim M_{2} \lesssim 0.7 M_{\odot}$ (with $M_{2}$ the mass of the donor star) respectively. A whole discussion of these systems can be found in Roberts (2013).

From an evolutionary point of view it is relevant to understand the processes that give rise to the formation of RB and BW systems. Shortly after the discovery of the first member of the group, PSR 1957+20, BWs have been considered as resulting from the evaporation of the donor star driven by pulsar emission. Regarding RBs, at present there are different proposals for their formation. Chen et al. (2013) considered CBS evolution with strong evaporation, assuming this to be possible because of geometrical effects. They presented a scenario in which BWs and RBs are consequence of different initial conditions. Benvenuto, De Vito, \& Horvath (2014) (BDVH14) considered CBS evolution including evaporation and irradiation feedback $a b$ initio (see below for a description). These 
phenomena lead to the occurrence of cyclic mass transfer (Büning \& Ritter 2004) when the system starts with $P_{\text {orb }}$ and $M_{2}$ in a range typical of RBs progenitors. They concluded that some RBs evolve to become BWs, while others should form MSP-helium white dwarf (He-WD) pairs. Smedlev et al. (2015) suggested that RBs are formed by the accretion induced collapse of oxygen/neon/magnesium WDs. The just-formed NS becomes a radio pulsar that irradiates and makes the donor star to undergo evaporation. They assumed that the turn-on of pulsar emission prevents further NS accretion and claim that in this way it is possible to account for the whole family of observed RBs.

In the recycled model of pulsars, the onset of accretion causes the NS to spin up to a hundreds of $\mathrm{Hz}$ and the NS mass to grow (see, e. g., Alpar et al. 1982). Standard CBSs evolution predicts a long-standing (order $10^{9}$ years) mass transfer episode in which the donor star loses about $70 \%$ of its mass. During this stage, the system is seen as an $\mathrm{X}$-ray source, and the pulsar remains hidden. The systems formed by a NS and a low-mass donor star with a mass $1.0 M_{\odot} \lesssim M_{2} \lesssim 3.5 M_{\odot}$, are known as Low-Mass X-Ray Binaries (LMXBs). After this long mass transfer episode, a few short-lived mass transfer events due to hydrogen thermonuclear flashes may occur. Very little amount of mass is exchanged in these episodes. The final state of the system is largely determined by the first mass transfer episode (see, e.g., Lin et al. 2011 and references therein).

A novel ingredient in the former LMXBs which should be important for relatively short orbital periods is the socalled irradiation feedback (Hameurv \& Ritter 1997). Here, irradiation feedback refers to the heating of the donor star by the luminosity produced by accretion onto the NS. It should affect the evolution of the companion and the whole spider system. Including this effect in evolutionary calculations leads to the occurrence of cyclic mass transfer episodes as described by Büning \& Ritter (2004) instead of the single long mass transfer episode cited above. Based on those calculations, we have argued for the existence of an evolutionary connection between BWs and RBs (see Figure 3 in BDVH14). Also, in this context evaporation means the process of removal of matter from the donor star due to pulsar emission.

In this paper we shall study the evolution of binary systems that lead to the formation of RBs in the theoretical framework provided by the models presented in BDVH14. More specifically, we are interested in the evolutionary state of the intriguing binary system containing PSR J1417-4402. The paper is organized as follow: Section 2 summarizes a few recent observations, in Section 3 we present the main characteristics of our code, in Section 4 we describe the calculations presented in this paper, and in Section 5 we present our main conclusions.

\section{SUMMARY OF RECENT OBSERVATIONS}

Three specific systems have been found giving support to the recycled scenario accepted to explain the millisecond pulsar formation. They behave as RBs switching between an accretion-disk state, with no radio pulsations (the LMXB state), and a rotation-powered state featuring radio pulsa- tions (the PSR state). These are know as transitional millisecond pulsars (tMSPs).

PSR J1023+0038, "the missing link", is a MSP with $P_{\text {spin }}=1.688 \mathrm{~ms}$ (where $P_{\text {spin }}$ is the spin period of the NS). The orbital period of the binary system is $P_{\text {orb }}=0.198 \mathrm{~d}$. It showed a typical behavior of an accretion state from May 2000 to December 2001, with clear evidence for the presence of an accretion disk. From 2002 it was in a quiescent state (Archibald et al. 2009). There is also evidence that its binary companion, with an estimated mass of $0.24 M_{\odot}$ (Deller et al. 2012), was filling its Roche lobe. Ten years later, Stappers et al. (2013) reported a change in its state; and did not detect the pulsar in their radio observations. Halpern et al. (2013) reported the characteristic doublepeaked $H \alpha$ line, indicative of the presence of an accretion disk. The system is presently again in the LMXB state (Patruno et al. 2014).

PSR J1824-2452I is another MSP in a spider system, located in the globular cluster M28. Its companion has a minimum mass of $M_{\min }=0.174 \pm 0.003 M_{\odot}$ (evaluated for a neutron star mass of $1.35 M_{\odot}$ and an inclination of the system of $90^{\circ}$, see Papitto et al. 2013). The orbital period of the system is $P_{\text {orb }}=0.4594 \mathrm{~d}$, and the pulsar spin $P_{\text {spin }}=3.93 \mathrm{~ms}$. Analysis of the records for this system reveals the short timescale of the switching mechanism between rotation and accretion powered states (PSR and LMXB states respectively). These changes occur in only few days to months, a lapse far smaller than the characteristic $\sim$ Gyr scale for the donor star evolution (Papitto et al. 2013).

The third member of this transitional group is XSS J12270-4859, initially classified as a LMXB with $P_{\text {orb }}=$ 0.2875 d. Bassa et al. (2014) presented radio, optical and Xray observations showing that XSS J12270-4859 is a LMXB that shifted to a radio MSP state between November 14 and December 21 of 2012. de Martino et al. (2015) presented an analysis of X-ray, UV and optical/near-IR photometric data obtained after the transition to a rotation-powered radio PSR state. Their analysis of the optical light curves give a mass ratio of $0.11 \lesssim q \lesssim 0.26$, whereby the mass of the donor star is found to be $0.15 M_{\odot} \lesssim M_{2} \lesssim 0.36 M_{\odot}$, considering a NS companion with $M_{\mathrm{NS}}=1.4 \underset{M_{\odot}}{ }$.

These three transitional systems clearly belong to the RB family.

On the characteristic observational plane used for studying the kind of CBSs related with recycled pulsars, namely the $M_{2}-P_{\text {orb }}$, RBs are located in a specific region, delimited by the orbital period value of the system and the estimated mass of the companion. However, a system that is outside the RB zone has been recently seen to undergo a transition from a LMXB to a PSR state, which is quite unexpected.

Abdo et al. (2010) discovered a $\gamma$-ray source, detected by the Large Area Telescope (LAT) on the Fermi Gammaray Space Telescope and first cataloged as 3FGL J1417.54402. Petrov et al. (2013) performed radio continuum observations with the Australian Telescope Compact Array, and reported no radio emission detected from the source (2012 September 20). In 2013 February 4, it was observed with the $6 \mathrm{~km}$ configuration and, again, no radio emission was detected. Strader et al. (2015) confirmed the nature of the optical counterpart, whose spectrum corresponds to a late $\mathrm{G}$ or early $\mathrm{K}$ star. Assuming the donor is filling its Roche 
lobe, they conclude that the object is a giant star. They further derived an orbital period of $P_{\text {orb }}=5.37385 \pm 0.00035 \mathrm{~d}$ and an eccentricity of $e=0.01 \pm 0.01$; the mass ratio for the system is $q=M_{2} / M_{\mathrm{NS}}=0.179 \pm 0.010$. The estimated masses for the components are $M_{\mathrm{NS}}=1.97 \pm 0.15 M_{\odot}$ and $M_{2}=0.35 \pm 0.04 M_{\odot}$. Camilo et al. (2016) confirmed the end of the LMXB stage when they found a $2.66 \mathrm{~ms}$ pulsar (PSR J1417-4402) around the 5.4 day optical variable discovered by Strader et al. (2015) as the companion star in the high-energy gamma-ray source 3FGL J1417.5-4402.

Camilo et al. (2016) attribute to eclipses the intervals in which the pulsar is not detected in their observations. Besides, they deduce that the delay in the pulse arrival, on at least two occations, is due to an ephemeral clump of ionized material.

The intrinsic temperature of the unheated companion star of PSR J1417-4402 deduced from the optical spectrum of is $T_{\text {eff }}=5000 \mathrm{~K}$ (Strader et al. 2015). Although some heating of the companion star photosphere by the pulsar wind should exist, the optical light curves show no clear evidence of this process, at least at a level of $0.1-0.2$ magnitudes. This may occur because the intrinsic luminosity of the giant/sub-giant component dominates the light curve. Camilo et al. (2016) assume an upper limit for the average temperature for the side facing the pulsar of $T_{\mathrm{h}}=5200 \mathrm{~K}$. Because of these observational facts, we shall consider that the temperature of the model should be in the interval $T_{\text {eff }}=5000-5200 \mathrm{~K}$.

The distance to PSR J1417-4402 was estimated by Strader et al. (2015), $d_{S}=4.4 \mathrm{kpc}$, based on the magnitude and optical spectrum of the companion and considering that it fill its Roche lobe. Camilo et al. (2016) estimate the distance from the radio pulsar dispersion measure (DM), using the distribution model of Cordes \& Lazio (2002), obtaining $d_{C}=1.6 \mathrm{kpc}$, and consider that the best DM-based distance estimation. But it is important to remark that PSR J1417-4402 has a high Galactic latitude, and the determination of DM could have uncertainties. Then $d_{S}$ can not be rouled out. Recently, a new model for the large-scale distribution of free electrons in the Galaxy was presented by Yao, Manchester, \& Wang 2017a) (know as YMW16 electron density model). The distance obtained based in this model is $2.16 \mathrm{kpc}$ (Yao, Manchester, \& Wang 2017b).

The typical $\mathrm{H} \alpha$ emission line with double-peaked morphology, indicative of the presence of an accretion disk, has been positively detected by Strader et al. (2015). These authors claim that the X-ray luminosity at $d_{S}$ is consistent with those of transitional MSPs in their disk states.

After a detailed analysis, Camilo et al. (2016) suggest that PSR J1417-4402 is in the radio ejection regime (see Section 3), and this scenario is favored by the orbital period value of this system. However, Archibald et al. (2015) detected coherent X-ray pulsations from the tMSP PSR J1023+0038 in its LMXB sate, interpreted as inflowing material heating the magnetic polar caps of the NS. This phenomena was seen in the other two know tMSPs, XSS J12270-4859 and IGR J18245-2452, as well as the tMSP candidate 1RXS J154439.4-112820. This highlights the complexity of the accretion scenario in this kind of systems (see Jaodand et al. (2016)).

In a previous paper (BDVH14) we have shown that RBs can experience transitions between LMXB and PSR states and vice versa. There is no theoretical reason to expect the occurrence of this behavior only for the RBs group (see Fig. 3 in BDVH14). Indeed, the zone of cyclic mass transfer episodes in the plane $M_{2}-P_{\text {orb }}$ covers a wide range of companion masses and orbital periods. Thus, systems like PSR J1417-4402 are naturally expected in the framework of our models.

With this perspective, we shall now attempt to characterize a possible progenitor range for the PSR J14174402 system, based on CBS calculations including irradiation feedback. In other words, we aim to clarify the evolutionary limits of the transitional systems and, as we shall see, this leads us to believe that should be some systems driven by the same evolution physics which lay outside the present bounds of the RBs group.

\section{THE CLOSE BINARY EVOLUTIONARY CODE}

We computed CBS evolution using our code (Benvenuto \& De Vito 2003), including irradiation feedback and evaporation. Whenever the pair is detached the code is fairly standard. When mass transfer conditions are attained it solves the structure of the donor, mass transfer rate and $P_{\text {orb }}$ implicitly. As in previous works, we assumed that the NS accretes a fixed fraction $\beta$ of the transferred matter up to the Eddington critical rate $\dot{\mathrm{M}}_{\mathrm{Edd}} \approx 2 \times 10^{-8} \mathrm{M}_{\odot} / \mathrm{y}$. Very low accretion rates $\left(\lesssim 1.3 \times 10^{-11} \mathrm{M}_{\odot} / \mathrm{y}\right)$ are prevented by the propeller mechanism (Romanova et al. 2008). Nevertheless, there are observations that show coherent X-ray pulsations at very low luminosities in PSR J1023+0038, that can be asociated to very low mass accretion onto the NS (see Archibald et al. 2015). Irradiation feedback has been included following Hameurv \& Ritter (1997). For a complete description see Benvenuto, De Vito, \& Horvath (2015). Our models also include evaporation due to pulsar emission. From an evolutionary point of view the latter represents a minor perturbation for the donor star at the stages studied in this paper.

It has been also been suggested (Ruderman, Shaham, \& Tavani 1989; Burderi et al. 2001) that the pressure due to radio pulsar emission may inhibit material lost by the donor star to be accreted by the NS. This phenomenon, usually called radio ejection may be relevant for spider systems. If radio ejection operates, it may inhibit the occurrence of irradiation feedback. Also, as we have commented above, in some cases observations indicate the presence of an accretion disk surrounding the NSs. van Haaften et al. (2012) studied the evolution of ultra-compact LMXBs, considering an accretion disk, allowing for the occurrence of short time scale disk instabilities, modulating the long term evolution computed here. Including radio ejection and/or accretion disks in our models is beyond the scope of this paper.

\section{RESULTS}

In order to find a plausible progenitor for the PSR J14174402 system, we explored the range of initial parameters 
with possibilities to reproduce the current state of that system. We based the exploration in our library of previous calculations (Benvenuto. De Vito, \& Horvath 2014), where we perfomed a detailed grid covering initial masses of the donor star between 1.00 and $3.50 M_{\odot}$, a $1.40 M_{\odot}$ NS companion, and initial orbital periods between 0.50 and 12 days. We find that a normal, solar composition donor star with an initial mass value of $M_{2}=2.5 M_{\odot}$ together with a NS of $M_{\mathrm{NS}}=1.4 M_{\odot}$ on a circular orbit with $P_{\text {orb }}=1.4$ days, and $\beta=0.50$ was our best candidate. In fact, as we shall show below, these initial conditions successfully reproduce the present state of the PSR J1417-4402 system. We considered different strengths for irradiation feedback controlled by the "coupling constant" $\alpha_{\text {irrad }}$. This parameter represents the fraction of the accretion luminosity released by the NS that effectively participates in the irradiation feedback process. Here we have considered $\alpha_{\text {irrad }}=0.00,0.01,0.10$ and 1.00. The main results of this paper are presented in Figs. 1 . 5.

When considering the case of PSR J1417-4402 system solely, it would be possible to argue that the donor star is indeed behaving as predicted by standard calculations and that the pulsar reveals itself due to accretion disk instabilities. However, the population of detected redbacks is rather numerous (see Fig. 4 of BDVH14), and it seems not plausible to attribute it only to disk instabilities (see, e.g., Hameury et al. 1998). Irradiated models depict the main characteristics of redback systems, especially the quasi Roche lobe overflow state showed by an important fraction of donor stars in this group of spiders. Besides, the whole spider evolution is nicely accounted for by irradiated models, like those presented in that paper and in previous calculations presented by our group (see, e.g., Benvenuto, De Vito, \& Horvath 2015). In the frame of irradiated models, LMXBs and transitional objects should correspond to systems undergoing mass transfer with the latter undergoing disk instabilities severe enough to eventually reveal the pulsar. Meanwhile, several pulsars should be observable due to cessation of mass transfer in each cycle. Certainly, to be conclusive in this point we would need to perform a population synthesis analysis, which is beyond the scope of this paper. We consider that irradiated models with cyclic mass transfer are more realistic than standard ones, and this should be valid for the case of PSR J1417-4402 system too.

In Figure 1 we show the Hertzsprung-Russell (HR) diagram for the cases of $\alpha_{\text {irrad }}=0.10$ and 0.01 in the upper and lower panels respectively, together with the results corresponding to non-irradiated model. Based on the range of estimated temperatures for the donor star $\log _{10}\left(T_{\text {eff }}\right)=$ $3.699 \lesssim \log _{10} T \lesssim \log _{10}\left(T_{h}\right)=3.716$ (Camilo et al. 2016), the pulsar companion is in an evolutionary state corresponding to the vertical zone of the right side in the HR diagram. This state is completely different from a WD. The He-WD state for the companion of PSR J14174402 was suggested by Camilo et al. (2016) based on the standard close binary evolution calculation performed by Podsiadlowski, Rappaport, \& Pfahl 2002). Moreover, the authors have difficulties trying to locate the companion star in this evolutionary state, because it is not clear if the latter is in a quasi-Roche lobe overflow (quasi-RLOF) or not (see Benvenuto, De Vito, \& Horvath 2015), if the mass transfer

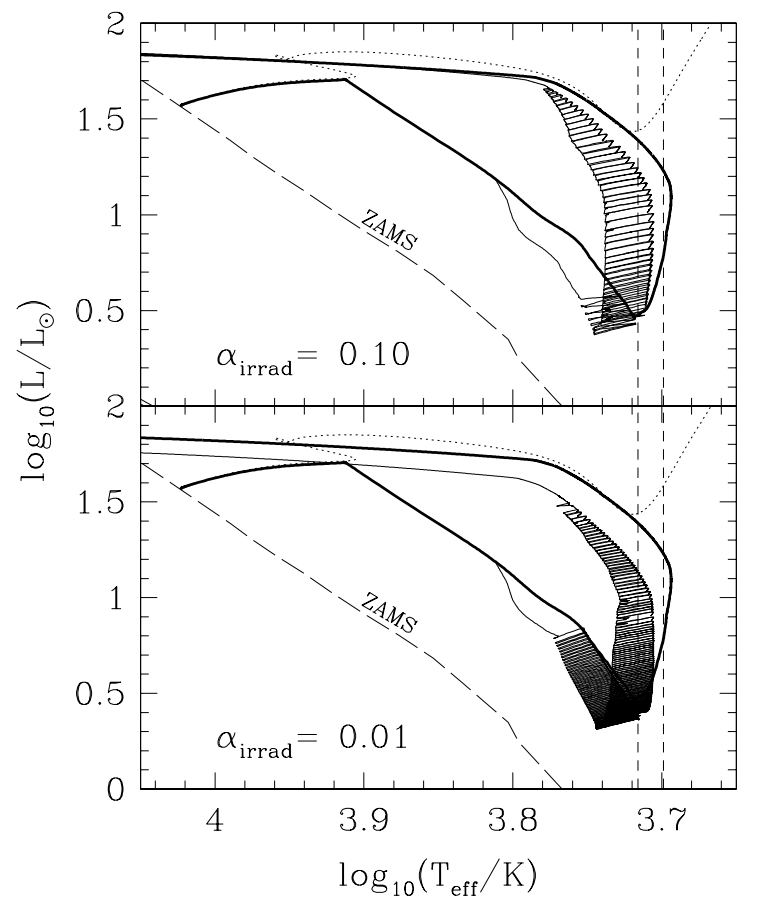

Figure 1. The evolution of a solar composition donor star in a spider system with initial $M_{2}=2.5 M_{\odot}, M_{\mathrm{NS}}=1.4, P_{\mathrm{orb}}=$ 1.4 days, and $\beta=0.50$. Upper and lower panels show the cases of $\alpha_{\text {irrad }}=0.10$ and 0.01 respectively. In both panels with a thick line we show the non-irradiated case $\left(\alpha_{\text {irrad }}=0.00\right)$ and the temperature range corresponding to this object (discussed in $\S$ (2), with vertical dash lines. Also in both panels, with dotted line we depict the evolution of an isolated star with the same mass and chemical composition and, with long dash line, the position of the zero age main sequence (ZAMS).

onto the NS has finished or not, or even if the pulsar is still ablating the companion. Nevertheless our model naturally fits the observed state of the companion of PSR J1417-4402.

As we can see from Figure 11 irradiated and nonirradiated models occupy the same place un the HR diagram. Nevertheless, irradiated models represent a large improvement in the physical description of these systems, since they naturally provide, e.g., the quasi-RLOF status observed in many redback systems.

The evolutionary stage we want to describe for this system is the PSR state. Observations show a little difference between the pulsar-irradiated and non-irradiated faces of the donor star. We could think of attributing this temperature difference to the homogenization of the residual heat that has remained in the envelope of the donor star from the LMXB phase, or due to the heating from the spin-down luminosity of the pulsar in this phase.

Regarding the response of the envelope to changes in the irradiation regime we should remark that these are quite fast. Let us consider the characteristic Kelvin-Helmholtz timescale $\tau_{K H}$ given in Hameury, King, \& Lasota (1986)

$\tau_{K H}=\frac{\rho C_{p} T H_{p}}{\phi_{*}}$ 
where $\rho$ is the density, $C_{p}$ is the specific heat at constant pressure, $T$ is the temperature, $H_{p}=-d r / d \ln P$ is the pressure scale height, and $\phi_{*}$ is the stellar flux. Applying this definition to the case of the our model for the present status of PSR J1417-4402, we found results very similar to those presented in Hameurv, King, \& Lasota (1986), in particular in the Figures $4 \mathrm{a}$ and $4 \mathrm{~b}$ of that paper. The outermost layers of the star react on very short timescales, far shorter than a year. Thus, the difference of 0.017 dex in the photospheric temperature of the irradiated and non-irradiated portions of the donor star should not be interpreted as a relic of a previous evolutionary stages in which the NS acted as a X-ray source but as a consequence of pulsar spin-down irradiation that is currently received by the star.

Let us estimate the luminosity received by the donor star to produce a 0.017 dex difference in the irradiated side. If $R$ is the radius of the donor star and $a$ is the orbital semiaxis, the irradiated and non-irradiated portions of its surface are $S_{i r r}=2 \pi R^{2}(1-R / a)$ and $S_{0}=2 \pi R^{2}(1+R / a)$ respectively. Then, the total luminosity of the donor is $L=2 \pi R^{2} \sigma\left[(1-R / a) T_{i r r}^{4}+(1+R / a) T_{0}^{4}\right]$, where $\sigma$ is the Stefan-Boltzmann constant and $T_{i r r}$ and $T_{0}$ are the temperatures at the irradiated and non-irradiated portions of the donor photosphere. If we define $\Delta L$ as the excess of luminosity emitted by the radiated portion of the donor, $\Delta L=2 \pi R^{2} \sigma(1-R / a)\left[T_{i r r}^{4}-T_{0}^{4}\right]$, and consider the case of PSR J1417-4402 where we have $R=4.8 R_{\odot}, R / a=0.24$, $\log T_{i r r}=3.716$, and $\log T_{0}=3.699, \Delta L=0.8359 L_{\odot}$.

Observed from the NS distance, the donor star represents a solid angle $\Delta \Omega=2 \pi\left[1-\sqrt{1-(R / a)^{2}}\right]$, that for PSR J1417-4402 is $\Delta \Omega=0.1836$. If for simplicity we consider isotropic emission, it corresponds to a pulsar luminosity of $L_{p s r}=57.2 L_{\odot}$. If we assume that this stems from the rotational energy $L_{p s r}=-2 \pi^{2} I_{N S} \dot{P} P^{-3}$ where $P$ is the pulsar period, $\dot{P}$ its time derivative and $I_{N S}$ is the moment of inertia of the NS. For PSR J1417-4402 $P=2.66 \mathrm{~ms}$, if we consider a typical value for the moment of inertia of $I_{N S}=10^{45} \mathrm{~g} \mathrm{~cm}^{2}$ we find $\dot{P}=2.06 \times 10^{-19} \mathrm{~s} / \mathrm{s}$ which is in the range of values observed for several millisecond pulsars. Considering this infered value for $\dot{P}$ we obtain a magnetic field strength of $7.5 \times 10^{8} \mathrm{G}$, using $B \simeq 3.2 \times 10^{19}(P[s] \dot{P}[s / s])^{1 / 2} G$ (Manchester \& Taylor 1977). This infered value is in the range corresponding to recicled pulsars $\sim 10^{8}-10^{9} \mathrm{G}$.

Figure 2 shows the mass transfer rate for the three sequences of irradiated models considered in this paper, and the non-irradiated model with a thick line. As we can see from this Figure, the model corresponding to very high irradiation $\alpha_{\text {irrad }}=1.00$ does not undergo cyclic mass transfer around of the mass value estimated for the companion of PSR J1417-4402. Thus, we rule out this extreme model as a possible progenitor for this system.

In Figure 3 we show the evolution of the mass ratio $q=M_{2} / M_{\mathrm{NS}}$ as a function of the the donor star mass, $M_{2}$. From this graph we conclude that our best candidate for the progenitor of the system containing PSR J1417-4402 is the corresponding to $\alpha_{\text {irrad }}=0.10$, since it is able to account for the correct values of $q$ and $M_{2}$, and therefore that of $M_{\mathrm{NS}}$.

In Figure 4 we show the evolution of the orbital period as a function of the donor star mass for the cases of $\alpha_{\text {irrad }}=0.00,0.01$ and 0.10. As we can see from this Figure, the progenitor with $\alpha_{\text {irrad }}=0.10$ also reproduces the appro-

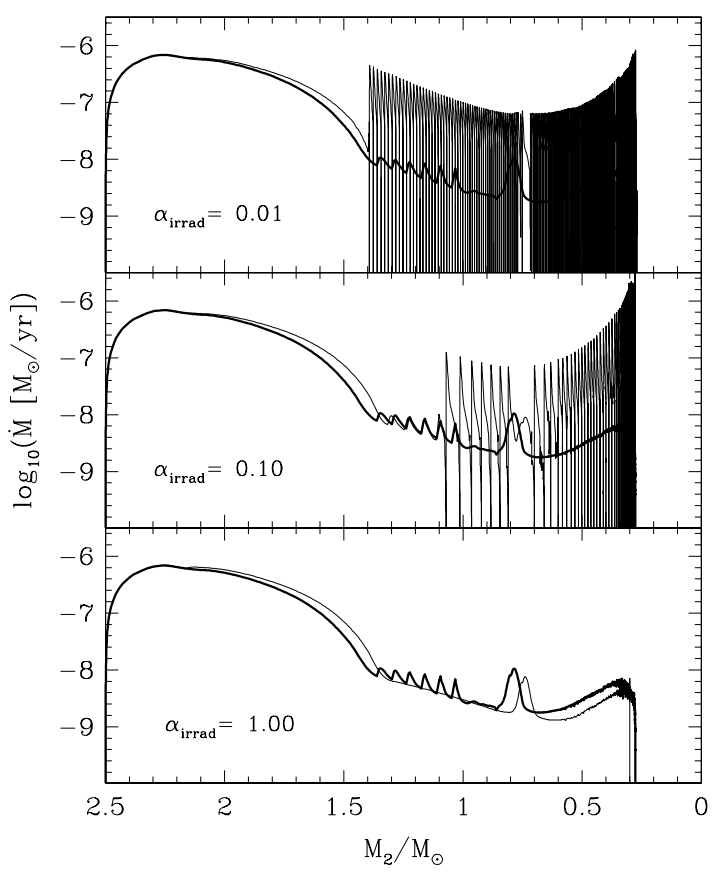

Figure 2. The mass transfer rate as a function of the donor mass for the four model sequences computed for this paper. We show the cases of $\alpha_{\text {irrad }}=0.01,0.10$ and 1.00 in the upper, middle and lower panels, respectively. In all panels, for comparison, we show with thick solid line the case of non-irradiated models.

priate value of the orbital period at the correct value of the donor star mass.

Finally, we show in Figure 5 the evolution of the ratio of the radii of the donor star to that of its equivalent Roche lobe as a function of its mass for $\alpha_{\text {irrad }}=0.10$ and 0.01 . As it stands, the donor star is in the quasi-RLOF state (Benvenuto, De Vito, \& Horvath 2015).

These results show that models considering irradiation feedback and evaporation are able to account for the main characteristics of the system containing PSR J1417-4402. Nevertheless, we want to remark that this solution is one of the possible ways to attain the current state of PSR J14174402. It represent the best solution based on our own hypothesis and calculations, but other simulations with different hypothesis and initial conditions could account for the evolution and the current state of this system as well.

\section{DISCUSSION AND CONCLUSIONS}

In this paper we have studied the possibility that the evolution of CBSs naturally accounts for the present status of the system containing PSR J1417-4402. For this purpose we have searched for systems that evolve to the masses and orbital period values deduced from observations. We have found that models with initial parameters $M_{\mathrm{NS}}=1.4 M_{\odot}$, $M_{2}=2.5 M_{\odot}, P_{\text {orb }}=1.4$ days, and $\beta=0.5$ evolve to get the required donor ans NS masses at the observed orbital 


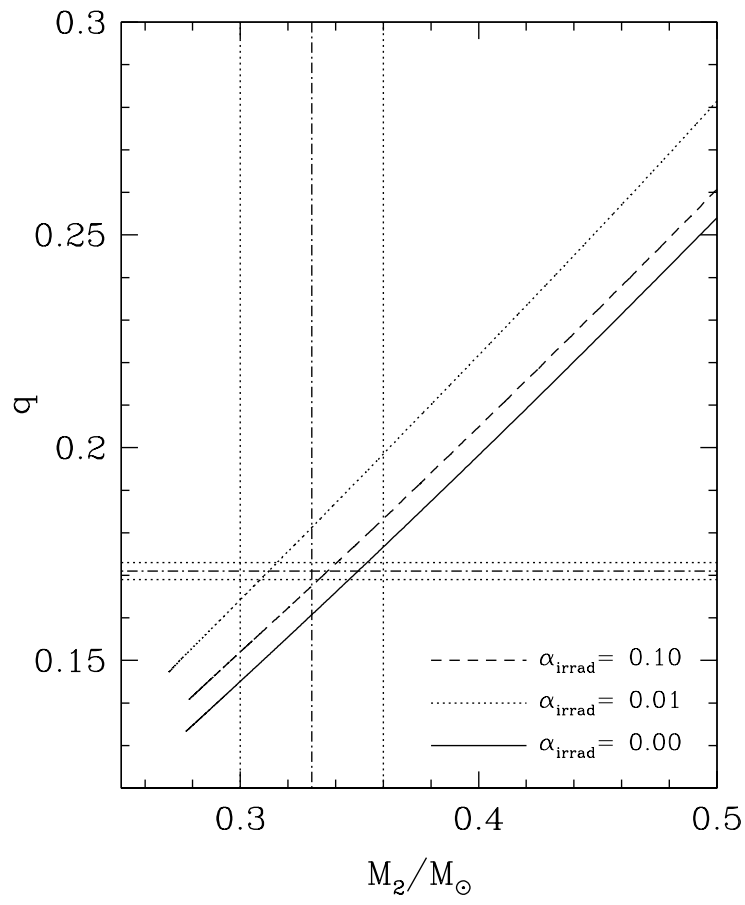

Figure 3. The mass ratio $q=M_{2} / M_{\mathrm{NS}}$ as a function of the donor star mass for the models presented in Fig. 1 With vertical and horizontal dot-dash lines we plot the estimated values of the donor mass and the mass ratio for the components of this system, respectively; with dot lines, we plot the errors in each of these estimated values. With full, dot-dash and short-dash line we depict the evolution of the mass ratio with the mass of the donor star for the cases of $\alpha_{\text {irrad }}=0.00,0.01$ and 0.10 , respectively.

period. For a positive detection of the pulsar emission, the system should be detached, a requirement fulfilled by models with $\alpha_{\text {irrad }}=0.10$, being in the quasi-RLOF state.

Regarding our assumption on the efficiency parameter $\beta$, we should remark that the value assumed in this paper is usually employed in the literature (see, e.g., Podsiadlowski, Rappaport, \& Pfahl 2002). However, it is possible (and even reasonable) that $\beta$ takes a different value and/or be variable depending on the evolutionary state of the spider system. In any case, we should stress that considering different values of $\beta$ will affect the initial values of the CBS suitable to account for this system, suggesting a degeneracy of the parameters leading to the desired state.

It is important to remark that while irradiation feedback is responsible for the occurrence of cyclic mass transfer episodes, evaporation is a necessary ingredient of the model, because of the observations of eclipses due to material present far beyond the Roche lobe of the donor star. In conditions of quasi-RLOF the donor star should be evaporating. In any case, the standard treatment of evaporation indicates a mass loss rate which is too low to cause a major effect on the evolution of the donor star at that stage. Also, as already stated, material lost from the system due to nonconservative mass transfer in previous RLOFs should also participate in the eclipses.

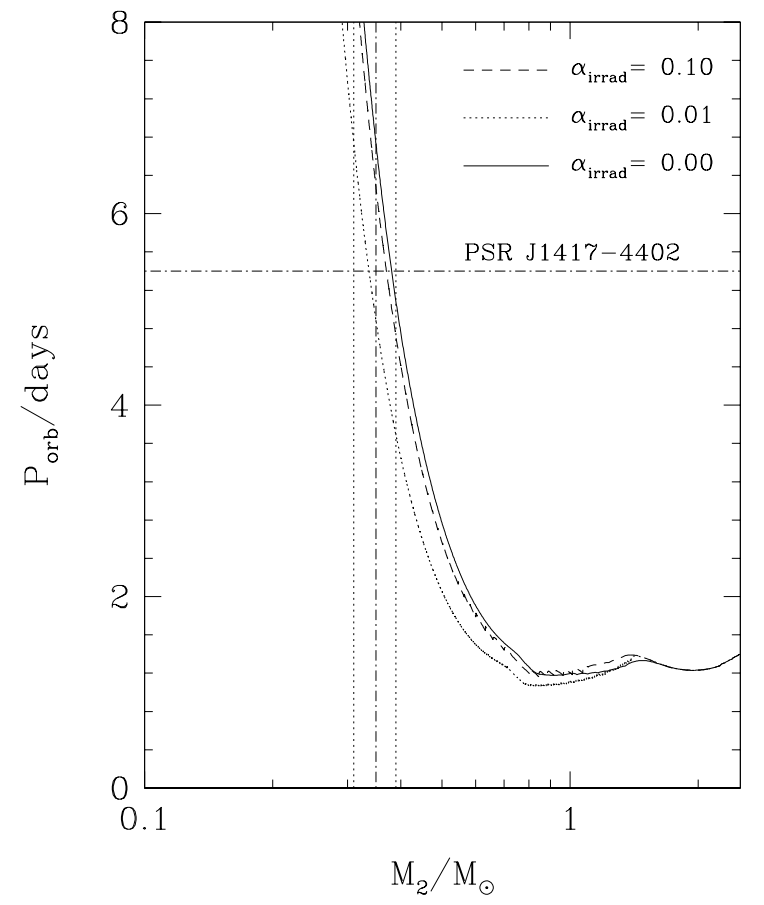

Figure 4. The evolution of the orbital period for the models presented in Fig. 1 as a function of the mass of the donor star for the same values of $\alpha_{\text {irrad }}$ showed in Figure 3 The lines have the same meaning as in that Figure. We indicate with horizontal dash-dot line the orbital period of the system. Also, with vertical dash-dot line we depict the mean value of the donor mass whereas dotted lines indicate the $1-\sigma$ error.

PSR J1417-4402 has an orbital period falling outside the parameter region usually considered for RBs. Current models indicate that the donor star of this system should evolve to become a He-WD. From a theoretical point of view and based in our irradiated models, the system not behave differently from those "standard RBs". Indeed, it can be checked that PSR J1417-4402 is located in the cyclic mass transfer region in Figure 3 of BDVH14. Therefore, based on our models with irradiation and evaporation, the observed behavior can be considered as expected.

The neighborhood of the position occupied by the companion of PSR J1417-4402 in the $M_{2}-P_{\text {orb }}$ plane is the region populated by CO-WDs companions. But, the companion of PSR J1417-4402 is clearly an extended, nondegenerate star (as the observations have shown, and has been depicted by our models). Then, we expect a mixed population of pulsars companions above of the usually considered redback region.

We want to thank our referee for his/her report, that lead us to fruitful discussions that contributed to largely improve our paper.

J.E.H. has been supported by Fapesp (São Paulo, Brazil) through the grant 2013/26258-4 and CNPq, Brazil funding agencies. 


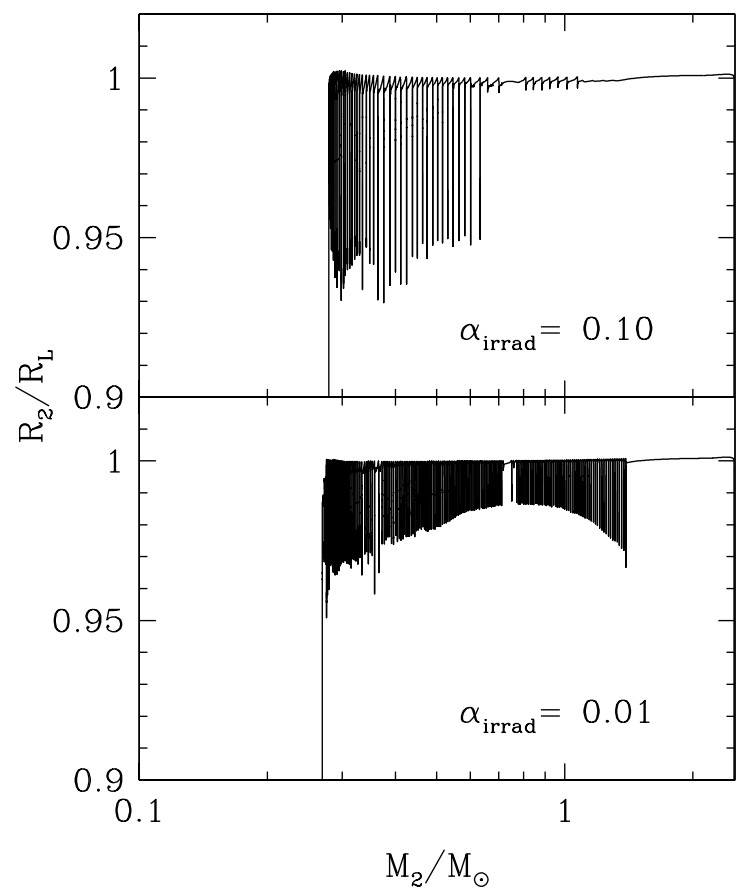

Figure 5. The radius of the donor star $R_{2}$, in units of the Roche lobe radius $R_{\mathrm{L}}$ as a function of the donor star mass for the models presented in Fig. 1 The donor star is in the RLOF or quasi-RLOF states.

\section{REFERENCES}

Abdo A. A., et al., 2010, ApJS, 188, 405

Alpar M. A., Cheng A. F., Ruderman M. A., Shaham J., 1982, Natur, 300, 728

Archibald A. M., et al., 2009, Sci, 324, 1411

Archibald A. M., et al., 2015, ApJ, 807, 62

Bassa C. G., et al., 2014, MNRAS, 441, 1825

Benvenuto O. G., De Vito M. A., 2003, MNRAS, 342, 50

Benvenuto O. G., De Vito M. A., Horvath J. E., 2014, ApJ, 786, L7 (BDVH14)

Benvenuto O. G., De Vito M. A., Horvath J. E., 2015, ApJ, 798,44

Büning A., Ritter H., 2004, A\&A, 423, 281647

Burderi L., et al., 2001, ApJ, 560, L71

Camilo F., et al., 2016, ApJ, 820, 6

Chen H.-L., Chen X., Tauris T. M., Han Z., 2013, ApJ, 775,27

Cordes J. M., Lazio T. J. W., 2002, astro, arXiv:astro-ph/0207156

Deller A. T., et al., 2012, ApJ, 756, L25

de Martino D., et al., 2015, MNRAS, 454, 2190

Fruchter A. S., Stinebring D. R., Taylor J. H., 1988, Natur, 333,237

Halpern, J.P., Gaidos,E., Sheffield, A., Price-Whelan, A.M., and Bogdanov, S.: 2013, The Astronomer's Telegram, 5514 .

Hameury J. M., King A. R., Lasota J. P., 1986, A\&A, 162, 71
Hameury J.-M., Ritter H., 1997, A\&AS, 123,

Hameury J.-M., Menou K., Dubus G., Lasota J.-P., Hure J.-M., 1998, MNRAS, 298, 1048

Jaodand A., Archibald A. M., Hessels J. W. T., Bogdanov S., D'Angelo C. R., Patruno A., Bassa C., Deller A. T., 2016, ApJ, 830, 122

Lin J., Rappaport S., Podsiadlowski P., Nelson L., Paxton B., Todorov P., 2011, ApJ, 732, 70

Manchester, R. N., Taylor, J. H. 1977. Pulsars. San Francisco: W. H. Freeman

Papitto A., et al., 2013, Natur, 501, 517

Patruno A., et al., 2014, ApJ, 781, L3

Petrov L., Mahony E. K., Edwards P. G., Sadler E. M., Schinzel F. K., McConnell D., 2013, MNRAS, 432, 1294

Podsiadlowski P., Rappaport S., Pfahl E. D., 2002, ApJ, 565,1107

Roberts M. S. E., 2013, IAUS, 291, 127

Romanova M. M., Kulkarni A. K., Long M., Lovelace R. V. E., 2008, AIPC, 1068, 87

Ruderman M., Shaham J., Tavani M., 1989, ApJ, 336, 507 Smedley S. L., Tout C. A., Ferrario L., Wickramasinghe D. T., 2015, MNRAS, 446, 2540

Stappers, B.W., Archibald, A., Bassa, C., Hessels, J., Janssen, G., Kaspi, V., Lyne, A., Patruno, A., and Hill, A.B.: 2013, The Astronomer's Telegram, 5513

Strader J., et al., 2015, ApJ, 804, L12

van Haaften L. M., Nelemans G., Voss R., Wood M. A., Kuijpers J., 2012, A\&A, 537, A104

Yao J. M., Manchester R. N., Wang N., 2017, ApJ, 835, 29

Yao J. M., Manchester R. N., Wang N., 2017, MNRAS, 468, 3289 